\title{
Psoriatic arthritis with hyperuricemia: more peripheral, destructive, and challenging to treat
}

\author{
L. Widawski $^{1} \cdot$ T. Fabacher $^{2} \cdot$ L. Spielmann ${ }^{1} \cdot$ JE. Gottenberg $^{3} \cdot$ J. Sibilia $^{3} \cdot$ PM. Duret $^{1} \cdot$ L. Messer $^{1,4} \cdot$ R. Felten $^{3}$
}

Received: 2 September 2021 / Revised: 7 January 2022 / Accepted: 10 January 2022 / Published online: 20 January 2022

(c) The Author(s) 2022

\begin{abstract}
Objective To study the impact of hyperuricemia on clinical presentation, severity, and associated comorbidities of psoriatic arthritis (PsA).

Methods Retrospective bicentric case-control study performed in Strasbourg and Colmar, France, from 2009 to 2019. Patients with PsA (according to ICD-10 coding) and at least one available serum urate (SU) measurement were included. Demographic, comorbidities, clinical, and radiographic data were collected. Hyperuricemia was defined as SU level $\geq 360 \mu \mathrm{mol} / \mathrm{L}$. Results We included 242 patients: 73 (30.2\%) had hyperuricemia and 15 (6.2\%) met 2015 ACR/EULAR criteria for gout. On univariate analysis, as compared with normo-uricemic patients, hyperuricemic patients were more frequently male $(72.6 \%$ vs $39.1 \%, p=1.6 \times 10^{-6}$ ) with higher body mass index ( $\left.30.9 \mathrm{vs} 28.7 \mathrm{~kg} / \mathrm{m}^{2}, p=0.015\right)$ and more comorbidities (Charlson comorbidity index: 2.6 vs $1.8, p=0.005)$. PsA started at an older age ( 47.5 vs 43 years, $p=0.016)$ was more polyarticular $(56.2 \%$ vs $41.9 \%, p=0.049)$ than axial $(9.6 \%$ vs $22.8 \%, p=0.019)$ and more destructive $(52.8 \%$ vs $37.4 \%, p=0.032)$. PsA patients with joint destruction more frequently had hyperuricemia than did others (37.6\% vs $25.8 \%, p=0.047)$. Multivariable analysis confirmed the association of hyperuricemic PsA with peripheral joint involvement (odds ratio 2.98; 95\% confidence interval $1.15-7.75 ; p=0.025)$ and less good response to treatment $(0.35 ; 0.15-0.87 ; p=0.024)$.

Conclusion Patients with hyperuricemic PsA show poorer response to PsA treatment and have more peripheral and destructive joint damage than normo-uricemic patients.
\end{abstract}

\section{Key Points}

- Gout and psoriatic arthritis (PsA) can co-exist in the same patient.

- Monosodium urate crystals might have a deleterious impact on PsA.

- Hyperuricemic PSA is more polyarticular, less frequently axial, and more destructive than normo-uricemic PsA.

- PsA with hyperuricemia should lead to more personalized medicine.

Keywords Crystal arthropathies · Gout · Hyperuricemia $\cdot$ Psoriatic arthritis $\cdot$ Psout

L. Messer and R. Felten contributed equally to this work.

\section{R. Felten}

renaud.felten@chru-strasbourg.fr

1 Rheumatology Department, Colmar Civil Hospital, 39 Avenue de la Liberté, 68024 Colmar Cedex, France

2 Department of Epidemiology and Public Health, Strasbourg University Hospital, 1 Place de l'Hôpital, 67000 Strasbourg, France

3 Rheumatology Department, Centre National de Référence des Maladies Auto-immunes Systémiques Rares RESO, Strasbourg University Hospital, 1 Avenue Molière, 67098 Strasbourg, France

4 UMR 7367, MISHA, Allée du Général Rouvillois, Dynamiques Européennes, 67083 Strasbourg Cedex, France

\section{Introduction}

Gout and psoriatic arthritis (PsA) are 2 common diseases that can co-exist in the same patient. These 2 diseases seem strongly linked, but the pathophysiological mechanisms of this link have not yet been defined. Nonetheless, monosodium urate (MSU) crystals play a pathogenic role in psoriasis with a mechanism we could extend to PsA [1]. We hypothesized that a new line of thinking regarding the convergence of gout and PsA in psout, involving MSU crystals, could prompt a potential new approach to treatment (urate-lowering therapy) among patients with active/refractory PsA. 
PsA and gout also share comorbidities, both associated with type 2 diabetes, high blood pressure, and increased risk of major adverse cardiovascular events (MACEs) [2, 3]. Metabolic syndrome is associated with both hyperuricemia and PsA [4, 5]. In a large cohort study of 37,315 patients followed for PsA, hyperuricemia was independently associated with cardiovascular disease in PsA patients [6].

Despite examples suggesting that hyperuricemia worsens and maintains skin psoriasis [7,8], it has not been shown to worsen PsA (leading to more severe, more erosive disease that is more resistant to treatments) or that hyperuricemia is associated with a particular clinical presentation of PsA. The literature contains few observational studies of the effect of hyperuricemia and/or gout on PsA $[5,9,10]$, and no study has accurately investigated its clinical presentation and severity. No prospective studies or clinical trials have examined hyperuricemia treatment in PsA. Moreover, we have no recommendations for treating isolated hyperuricemia. Therefore, in the absence of a gout attack history, a patient, even with PsA, should not receive hypo-uricemic therapy $[11,12]$.

This study aimed to compare PsA patients with normouricemia and hyperuricemia. Our main objective was to assess the impact of hyperuricemia on PsA in its clinical presentation, its severity, and associated comorbidities.

\section{Methods}

This was a retrospective case-control, bicentric cohort study. Patients from Strasbourg University Hospital and Colmar Civilian Hospital, France, were included from January 1, 2009, to December 20, 2019.

We included patients with a history of PsA according to their rheumatologists and codes of International Classification of Diseases, 10th edition (ICD-10) and the presence of at least one serum urate (SU) measurement in the medical file. In case of diagnostic doubt with rheumatoid arthritis (e.g., presence of anti-CCP antibodies or rheumatoid factor) during the medical file review, patients were excluded if they met the 2010 ACR/EULAR classification criteria for rheumatoid arthritis.

We excluded patients who expressed their opposition to participate to the study or with a diagnosis other than PsA after the medical file review.

Patients with normo-uricemia and hyperuricemia were compared according to a hyperuricemia threshold $\geq 360 \mu \mathrm{mol} / \mathrm{L}$, whether they were women or men, based on the median SU level for each patient. To minimize the impact of one isolated hyperuricemia event (e.g., due to an acute renal failure) and to promote the inclusion in the hyperuricemic group with persistent and lasting hyperuricemia, we used the median value for each patient to determine whether they were above this cutoff or not. The median was used to lower sensitivity to extreme values and because of the distribution of these values. This $360-\mu \mathrm{mol} / \mathrm{L}$ cutoff was chosen for several reasons. In vitro, MSU crystals crystallize at $35^{\circ} \mathrm{C}$ above the threshold of $360 \mu \mathrm{mol} / \mathrm{L}$, at which the risk of gout attack is increased in both women and men [13, 14]. The 2015 ACR/EULAR criteria for gout indicate this threshold of $360 \mu \mathrm{mol} / \mathrm{L}$ $(60 \mathrm{mg} / \mathrm{L})$, and no point is awarded below this threshold, regardless of sex [15]. Uricemia is an unsteady variable, depending on several factors including renal function [16]. A patient with normo-uricemia may experience hyperuricemia in the event of acute renal failure. To not include the patient in the wrong group (normo-uricemic vs hyperuricemic PsA), we excluded all patients who had hyperuricemia on the basis of a single SU test and who had acute renal failure at the time of the SU test.

Demographic, clinical, biological, and radiological available data were collected. Data collected for PsA included disease duration, age at PsA onset, HLA-B27 status, characteristics of clinical manifestations, and therapeutic history (including conventional synthetic disease-modifying antirheumatic drugs [csDMARDs], biologic DMARDs, and targeted synthetic DMARDs). PsA was defined for each patient according to the five Moll and Wright subtypes: oligoarticular, polyarticular, axial, distal, and mutilant [17]. Coexistence of several subtypes was possible. A "good response to ongoing PsA treatment" was defined by the absence of joint flare and elevated CRP level $(<4 \mathrm{mg} / \mathrm{L})$ at the last medical follow-up and if the PsA treatment (csDMARDs, bDMARDs, and/or tsDMARDs) remained unchanged at the last medical appointment. The absence of joint flare was defined by the absence of painful or swelling joint(s) based on clinical examination and/or ultrasonography exploration by the rheumatologist. We were not able to assess PsA with a disease activity index because of the retrospective design of our study. Regarding psoriasis, clinical features of the manifestation and therapeutic history (including psoralene ultraviolet A [PUVA] therapy) were collected.

For each patient, every SU measurement available was collected, as was the number of tests reported, in order to calculate the median SU level for each patient. A history of gout attack or evidence of MSU crystals within a joint was collected. Each patient was tested for 2015 American College of Rheumatology/European League Against Rheumatism (ACR/EULAR) gout criteria. Data on hypo-uricemic and gout attack treatment were collected.

Radiologic characteristics of PSA and gout from standard X-rays, ultrasonography, tomodensitometry, and MRI were collected. PsA was defined as destructive with any erosion related to PsA regardless of the technique (X-ray, 
ultrasonography, MRI, or CT scan). In addition to comparing hyperuricemic and normo-uricemic patients, we compared patients with destructive and non-destructive PSA.

We collected data on cardiovascular comorbidities, metabolic syndrome (according to the new International Diabetes Federation definition [18]), MACEs, family history of MACEs, history of diabetes, history of moderate to severe chronical renal failure (estimated glomerular filtration rate $<60 \mathrm{~mL} / \mathrm{min}$ ), history of non-alcoholic steatohepatitis, and sleep apnea syndrome. The Charlson comorbidity index was calculated [19]. Toxic exposures such as active smoking and alcohol consumption ( $>2$ units of alcohol/day) were collected.

In the descriptive part of the analysis, all data collected were summarized as number (percentage) for categorical data and mean (SD), median, and interquartile range (Q1-Q3) for quantitative data. For univariate analysis, the Fisher or chi-square test was used to compare categorical data and Wilcoxon or Student $t$ test for quantitative data. The Shapiro test associated with data visualization was used to assess normality. We did not calculate the sample size needed because we included all available patients. We used logistic regression to analyze the primary outcome with estimation of odds ratios (ORs) and 95\% confidence intervals (CIs). Model included 7 "hyperuricemia" variables, which were selected according to expert knowledge from the literature, univariate analysis, and backward stepwise selection on the Akaike Information Criterion [20]. To evaluate the impact of hyperuricemia on PsA in its clinical presentation, its severity, and associated comorbidities, we used the same methodology. Goodness of fit of the model was assessed with the Hosmer-Lemeshow goodness-of-fit test. Missing data were assigned by multiple imputation if they did not concern more than $30 \%$ of the variable's data; otherwise, the variables were excluded from the model. $p<0.05$ was considered statistically significant. All analyses involved using R 4.0.

This study was validated on August 24, 2020, by the Strasbourg University Hospital Ethics Committee (CE2020-134). Data were anonymized, and then recorded in an Excel table. An anonymous number was established for each patient so that data shared between investigators remained anonymous. Data were stored and analyzed with respect to the rights of all patients.

\section{Results}

We excluded 26 patients after reading the medical file because they did not present PsA. Ten patients had rheumatoid arthritis, 6 exclusives axial spondyloarthritis without psoriasis, 2 SAPHO syndrome, 2 an isolated dactylitis episode without any other articular or extra-articular sign,
3 fibromyalgia without any inflammatory rheumatism, and 1 each isolated osteoarthritis, isolated gout, and isolated skin psoriasis. Within the rheumatology department of the 2 hospitals, 268 patients met our inclusion criteria: 168 in Strasbourg and 74 in Colmar.

The main characteristics of the cohort are in Table 1 . The mean age was 58.2 (SD 12.8) years; $50.8 \%$ were women; and patients were overweight (mean body mass index [BMI] 29.3 [SD 5.9] $\left.\mathrm{kg} / \mathrm{m}^{2}\right)$. In total, $34(30.6 \%)$ patients were HLAB27-positive. The median duration of PsA was 11 [Q1-Q3 6-19] years, and mean age at PsA onset was 44.4 (SD 14) years. Overall, 99 (42.1\%) patients had radiographic destructive involvement related to PsA, and 158 (80.3\%) showed good response to the last ongoing treatment. As expected, most patients had peripheral articular involvement and 45 (19\%) had isolated axial involvement. The mean SU level was 309.2 (SD 88.04) $\mu \mathrm{mol} / \mathrm{L}$. Three (1.2\%) patients had a history of MSU crystals in synovial fluid, 15 (6.2\%) had a history of gout attack, and $15(6.2 \%)$ met the 2015 ACR/ EULAR criteria for gout.

The comparison between patients with hyperuricemia $(n=73)$ and normo-uricemia $(n=169)$ is in Table 2. As compared with normo-uricemic patients, hyperuricemic patients were older (mean 61 vs 57 years, $p=0.020$ ) and more frequently men $(72.6 \%, n=53$, vs $39.1 \%, n=66$, $\left.p=1.6 \times 10^{-6}\right)$. They had higher mean BMI ( 30.9 vs $28.7 \mathrm{~kg} /$ $\left.\mathrm{m}^{2}, p=0.015\right)$. The 2 groups did not differ in HLA-B27 positivity. The mean age at PsA onset was significantly higher in the hyperuricemic than normo-uricemic group: 47.5 (SD $13.71)$ vs 43 (SD 14) years ( $p=0.016$ ), but the groups did not differ in median duration of PsA. At the end of followup, as compared with normo-uricemic patients, hyperuricemic patients had a more peripheral PsA involvement (89\%, $n=65$, vs $76.7 \%, n=128, p=0.033$ ) and more polyarticular disease $(56.2 \%, n=41$, vs $41.9 \%, n=70, p=0.049)$. They had significantly less isolated axial involvement of PsA (9.6\%, $n=7$, vs $22.8 \%, p=0.019)$. Overall, $38(52.8 \%)$ had radiographic destructive PsA as compared with 63 (38.7\%) of normo-uricemic patients $(p=0.047)$. Erosion seen on standard X-rays was more frequent for hyperuricemic than for normo-uricemic patients $(43.7 \%, n=31$, vs $28 \%, n=44$, $p=0.023$ ). Good response to ongoing PsA treatment was lower for hyperuricemic than normo-uricemic patients (70.7\%, $n=41$, vs $84.2 \%, n=117, p=0.048)$, with no difference between the groups in distribution of last ongoing treatments.

The study of the main comorbidities is in Table 3. The mean Charlson comorbidity index was significantly higher for hyperuricemic than normo-uricemic patients (2.6 vs $1.8, p=0.005)$. Significantly more hyperuricemic than normo-uricemic patients had metabolic syndrome (53.4\%, $n=39$, vs $32.5 \%, n=55, p=0.003$ ), high blood pressure $\left(57.5 \%, n=42\right.$, vs $\left.29.6 \%, n=50, p=5.3 \times 10^{-5}\right)$, MACEs 
Table 1 Characteristics of the cohort $(n=242)$

\begin{tabular}{|c|c|c|}
\hline Variables & $n(\%)$ & Median (Q1-Q3) \\
\hline Age (years) & & $58(50-67)$ \\
\hline Women & $123(50.8 \%)$ & \\
\hline Men & $119(49.2 \%)$ & \\
\hline BMI $\left(\mathrm{kg} / \mathrm{m}^{2}\right)$ & & $28.78(25.3-32.9)$ \\
\hline HLA-B27-positive & $34(30.6 \%)$ & \\
\hline Age at PsA onset (years) & & $45.5(35-53)$ \\
\hline Duration of PsA evolution (years) & & $11(6-19)$ \\
\hline Peripheral PsA & $193(80.4 \%)$ & \\
\hline Isolated peripheral PsA & $141(58.7 \%)$ & \\
\hline Oligoarticular PsA subset & $82(34.2 \%)$ & \\
\hline Polyarticular PsA subset & $111(46.2 \%)$ & \\
\hline Isolated axial PsA subset & $45(18.7 \%)$ & \\
\hline History of dactylitis & $65(26.9 \%)$ & \\
\hline History of enthesitis & $35(14.5 \%)$ & \\
\hline Radiographic destructive PsA & $99(42.1 \%)$ & \\
\hline Positive response to PsA CASPAR criteria & $237(98.3 \%)$ & \\
\hline No treatment & $31(13 \%)$ & \\
\hline NSAIDs alone & $7(2.9 \%)$ & \\
\hline Apremilast alone & $9(3.8 \%)$ & \\
\hline csDMARDs alone & $71(29.5 \%)$ & \\
\hline bDMARDs alone & $54(22.3 \%)$ & \\
\hline csDMARDs + bDMARDs & $62(25.6 \%)$ & \\
\hline Good response to last ongoing treatment & $158(80.2 \%)$ & \\
\hline Number of SU measurements & & $2(1-3)$ \\
\hline Minimal SU level & & $276(224.25-339.8)$ \\
\hline Maximal SU level & & $329.5(263.75-397.5)$ \\
\hline Median SU level & & $300.25(246.75-374.4)$ \\
\hline Median SU level $\geq 360 \mu \mathrm{mol} / \mathrm{L}$ & $73(30.2 \%)$ & \\
\hline Median SU level $\geq 420 \mu \mathrm{mol} / \mathrm{L}$ & $19(7.9 \%)$ & \\
\hline Evidence of MSU crystals (joint) & $3(1.2 \%)$ & \\
\hline History of gout attack & $15(6.2 \%)$ & \\
\hline Positive response to 2015 ACR/EULAR gout criteria & $15(6.2 \%)$ & \\
\hline
\end{tabular}

$Q 1-Q 3$, quartile 1 to 3 ; BMI, body mass index; NSAIDs, non-steroidal anti-inflammatory drugs; $c s D$ $M A R D s$, conventional synthetic disease-modifying anti-rheumatic drugs; bDMARDs, biologic diseasemodifying anti-rheumatic drugs; $P S A$, psoriatic arthritis; $S U$, serum urate; $M S U$, monosodium urate; $C A S$ $P A R$, classification of psoriatic arthritis; ACR/EULAR, American College of Rheumatology/European League Against Rheumatism
( $16 \%, n=12$ vs $5 \%, n=8, p=0.004)$, ischemic stroke $(8.2 \%, n=6$, vs $1.8 \%, n=3, p=0.024)$, acute coronary syndrome $(9.6 \%, n=7$, vs $3 \%, n=5, p=0.048)$, moderate or chronic renal failure $(15.1 \%, n=411$, vs $3 \%, n=5$, $p=0.001)$, and type 2 diabetes $(30.1 \%, n=22$, vs $14.2 \%$, $n=24, p=0.007$ ).

Uricemia was measured more frequently in patients with destructive than non-destructive PsA (mean 4.66 [SD 7.29] vs 3.25 [SD 5.49], $p=0.024$ ) and the median SU level was significantly higher (median 321 [Q1-Q3 268-382] vs 288.8 [234-359.1] $\mu \mathrm{mol} / \mathrm{L}, p=0.0038)$. Destructive disease was associated with SU level $\geq 300 \mu \mathrm{mol} / \mathrm{L}(59 \%, n=60$, vs $45 \%$ non-destructive disease, $n=60, p=0.037)$ and $\geq 360 \mu \mathrm{mol} / \mathrm{L}$ (37.6\%, $n=38$ vs $25.8 \%, n=34, p=0.047)$.

On multivariable analysis based on the "hyperuricemia" variable (Table 4), variables significantly associated with hyperuricemia in PsA were male sex, high blood pressure, moderate or severe chronic renal failure, PUVA therapy, peripheral PsA involvement, and poor response to PsA ongoing treatment. Probability of peripheral PsA with hyperuricemia was increased threefold (OR 2.98, 95\% CI $1.15-7.75, p=0.025)$. The probability of a good response to ongoing treatment was reduced for patients with PsA and hyperuricemia (OR 0.35, 95\% CI 0.15-0.87, $p=0.024)$. 
Table 2 Univariate analysis: principal characteristics of normo-uricemic and hyperuricemic patients with psoriatic arthritis (PsA)

\begin{tabular}{|c|c|c|c|c|c|c|}
\hline \multirow[t]{2}{*}{ Variables } & \multicolumn{2}{|c|}{ Normo-uricemic $(n=169)$} & \multicolumn{3}{|c|}{ Hyperuricemic $(n=73)$} & \multirow[t]{2}{*}{$p$} \\
\hline & $n(\%)$ & Median (Q1-Q3) & $n(\%)$ & Median (Q1-Q3) & & \\
\hline Age (years) & 169 & $57(48-66)$ & 73 & $61(53-68)$ & & $\mathbf{0 . 0 2 0}$ \\
\hline Female & $103(61 \%)$ & & $20(27.4 \%)$ & & & $1.6 \times 10^{-6}$ \\
\hline Male & $66(39.1 \%)$ & & $53(72.6 \%)$ & & & \\
\hline BMI $\left(\mathrm{kg} / \mathrm{m}^{2}\right)$ & 148 & $28.3(24.3-32.2)$ & 64 & $30(26.3-34.5)$ & & 0.015 \\
\hline \multicolumn{7}{|l|}{ Psoriatic arthritis } \\
\hline HLA-B27-positive & $24(32 \%)$ & & $10(27.8 \%)$ & & & 0.83 \\
\hline Age at PsA onset (years) & 164 & $44.5(32.8-52)$ & 72 & & $49(39.5-57.3)$ & 0.016 \\
\hline Duration of PsA evolution (years) & 164 & $11(6-19)$ & 72 & & $11(8-17)$ & 0.85 \\
\hline Peripheral PsA & $128(76.6 \%)$ & & $65(89 \%)$ & & & $\mathbf{0 . 0 3 3}$ \\
\hline Oligoarticular PsA subset & $58(34.7 \%)$ & & $24(32.9 \%)$ & & & 0.88 \\
\hline Polyarticular PsA subset & $70(41.9 \%)$ & & $41(56.2 \%)$ & & & 0.049 \\
\hline Isolated axial PsA subset & $38(22.8 \%)$ & & $7(9.6 \%)$ & & & 0.019 \\
\hline History of dactylitis & $42(25.1 \%)$ & & $23(31.5 \%)$ & & & 0.34 \\
\hline History of enthesitis & $21(12.5 \%)$ & & $14(19.2 \%)$ & & & 0.23 \\
\hline Radiographic destructive PsA & $61(37.4 \%)$ & & $38(52.8 \%)$ & & & $\mathbf{0 . 0 3 2}$ \\
\hline \multicolumn{7}{|l|}{ Last ongoing treatment for PsA } \\
\hline No treatment & $24(14.5 \%)$ & & $7(9.6 \%)$ & & & 0.40 \\
\hline NSAID alone & $4(2.4 \%)$ & & $3(4.1 \%)$ & & & 0.44 \\
\hline Apremilast alone & $4(2.4 \%)$ & & $5(6.9 \%)$ & & & 0.14 \\
\hline csDMARD alone & $48(28.6 \%)$ & & $23(31.5 \%)$ & & & 0.65 \\
\hline bDMARD alone & $40(23.7 \%)$ & & $14(19.2 \%)$ & & & 0.50 \\
\hline csDMARD + bDMARD & $40(23.7 \%)$ & & $22(30.1 \%)$ & & & 0.34 \\
\hline Good response to last ongoing treatment & $117(84.2 \%)$ & & $41(70.7 \%)$ & & & 0.048 \\
\hline \multicolumn{7}{|l|}{ Uricemia and gout } \\
\hline Median SU level & 169 & $274(227-305)$ & 73 & & $398(380-420)$ & $5.5 \times 10^{-5}$ \\
\hline Evidence of MSU crystals & $0(0 \%)$ & & $3(4.1 \%)$ & & & $\mathbf{0 . 0 2 7}$ \\
\hline History of gout attack & $2(1.2 \%)$ & & $13(17.8 \%)$ & & & $4.6 \times 10^{-6}$ \\
\hline $\begin{array}{l}\text { Positive response to } 2015 \text { ACR/EULAR } \\
\text { gout criteria }\end{array}$ & $1(0.6 \%)$ & & $14(19.2 \%)$ & & & $2.3 \times 10^{-7}$ \\
\hline
\end{tabular}

Q1-Q3, quartile 1 to 3; BMI, body mass index; NSAIDs, non-steroidal anti-inflammatory drugs; csDMARDs, conventional synthetic diseasemodifying anti-rheumatic drugs; $b D M A R D s$, biologic disease-modifying anti-rheumatic drugs; PsA, psoriatic arthritis; $S U$, serum urate; $M S U$, monosodium urate; CASPAR, classification of psoriatic arthritis; ACR/EULAR, American College of Rheumatology/European League Against Rheumatism

Bold: $p<0.05$

Radiographical destructive PsA was associated but not significantly with hyperuricemia (OR 1.45, 95\% CI 0.71-2.96, $p=0.31$.

\section{Discussion}

As compared with normo-uricemic patients, hyperuricemic patients were more frequently male, with a more polyarticular and less axial PsA presentation, a more destructive radiographic involvement, more cardiovascular and renal comorbidities, and poorer response to PsA treatment. In addition, median SU level was higher with destructive than non-destructive PSA. Moreover, SU level was measured more frequently in patients with destructive than non-destructive PSA. These latter data may be explained by more frequent PsA flares with destructive than nondestructive PSA and more frequent measurement of SU level in this case to search for a differential diagnosis such as gout.

Our cohort of patients with PsA is mainly comparable to the known epidemiology of PsA [2, 21-23]. There were some notable differences: $46 \%$ of our patients had polyarticular PsA as compared with $20 \%$ in the literature [2]. This situation could be explained by an inclusion bias because all our patients were tested for uricemia in that a polyarticular 


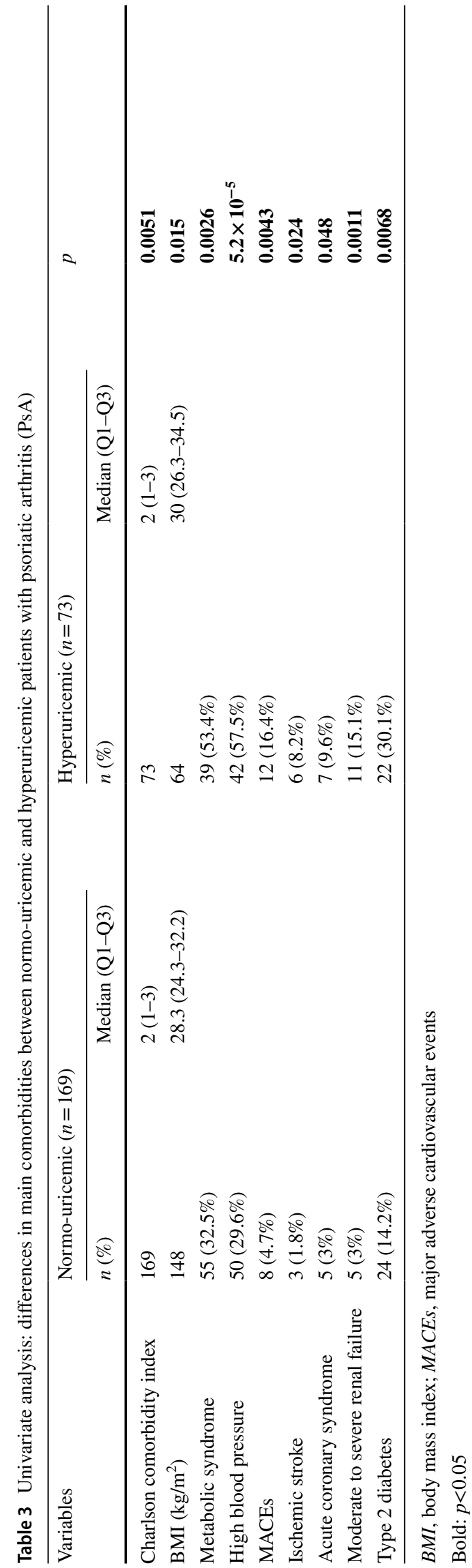

Table 4 Multivariable analysis of variables associated with hyperuricemia in PsA

\begin{tabular}{llll}
\hline Variable & $O R$ & $95 \% C I$ & $p$ value \\
\hline Male sex & 3.78 & $1.79-8.03$ & $\mathbf{0 . 0 0 0 6}$ \\
High blood pressure & 2.28 & $1.17-4.46$ & $\mathbf{0 . 0 1 6}$ \\
Chronic renal failure & 7.15 & $1.83-27.87$ & $\mathbf{0 . 0 0 4 8}$ \\
PUVA therapy & 2.75 & $1.06-7.12$ & $\mathbf{0 . 0 3 7}$ \\
Peripheral PsA & 2.98 & $1.15-7.75$ & $\mathbf{0 . 0 2 5}$ \\
Good response to ongoing treatment & 0.35 & $0.15-0.87$ & $\mathbf{0 . 0 2 4}$ \\
Radiographic destructive PsA & 1.45 & $0.71-2.96$ & 0.31
\end{tabular}

$O R$, odds ratio; $95 \% C I, 95 \%$ confidence interval; $P S A$, psoriatic arthritis; PUVA, psoralene ultraviolet A

Bold: $p<0.05$

presentation could suggest the diagnosis of gout. Only $14.5 \%$ of our patients had enthesitis (vs 30 to $50 \%$ in the literature), and $26 \%$ had dactylitis (vs 40 to $50 \%$ in the literature) [2]. This finding could be explained by the retrospective nature of our study, which may lead to lack of clinical data. There are no detailed data on the frequency of erosive PsA. The severity of PsA may have been overestimated in our cohort because of the inclusion criteria in our tertiary referral hospitals. Our rate of destructive involvement of PsA reached $42 \%$, which is comparable to a recent Italian study (34.7\% of 492 patients) [24].

The prevalence of gout in the French general population is $0.9 \%$ [25] and was $4.9 \%$ for men and $1.2 \%$ for women in a US cohort [10]. In our cohort of patients with PsA, the prevalence was $6.2 \%$ (only men), which is higher than in the general population. A few reports described patients with gout and psoriasis [26-29] and the coexistence of gout and PsA [27, 30-32]. These studies highlight the many common, sometimes confusing, risk factors shared by gout and PsA.

The prevalence of hyperuricemia in Europe reaches up to $25 \%$ [33]. In our PsA cohort, this prevalence was 30\%. These data are similar to the $30.6 \%$ prevalence of PsA in a Chinese cohort [9] and the $31.9 \%$ prevalence in a prospective Canadian cohort [5]. In contrast, in a Japanese case-control study, the prevalence of hyperuricemia in PsA patients was only $22 \%$ [34]. However, SU level thresholds were not the same in all of these studies; hyperuricemia was defined as above $360 \mu \mathrm{mol} / \mathrm{L}$ for women and $420 \mu \mathrm{mol} / \mathrm{L}$ for men in the Chinese study, above $360 \mu \mathrm{mol} / \mathrm{L}$ for women and $450 \mu \mathrm{mol} / \mathrm{L}$ for men in the Canadian study, and above $330 \mu \mathrm{mol} / \mathrm{L}$ for women and $468 \mu \mathrm{mol} / \mathrm{L}$ for men in the Japanese study. In our study, statistical analyses were performed with different SU level thresholds, including $360 \mu \mathrm{mol} / \mathrm{L}$ for women and $420 \mu \mathrm{mol} / \mathrm{L}$ for men. The findings remained the same, but the small number of patients in our cohort with median SU level $>420 \mu \mathrm{mol} / \mathrm{L}$ would have implied lack of power in statistical analysis. 
Little is known about the stability of the measurement in the same person over time, and some studies are limited because they measured SU level at a single time point [35]. However, SU level may vary depending on several factors [16, 36-38]. To overcome this eventual bias, we used the median of all SU measures for each patient. Indeed, we did not want to classify as "hyperuricemic" a patient who would have repeated normal SU levels but an unusual single high level, which could have been linked to confusing factors. As an example, kidney failure, both acute [39] and chronic [40], is known to be associated with hyperuricemia and gout, regardless of which is the cause or consequence. To avoid this "renal" bias, the presence or not of co-existing acute renal impairment was specified if only one uricemia sample was available and was $\geq 360 \mu \mathrm{mol} / \mathrm{L}$. Moderate or severe chronic renal failure was included in both univariate and multivariable analyses. Another obvious example could be the history of hypo-uricemic treatment. In our cohort, 3 of 14 patients with a history of treatment with allopurinol belonged to the normo-uricemic group because their median SU level was above the threshold. One had a history of gout attack and may have been misclassified. The 2 others received treatment for only isolated hyperuricemia; we did not exclude them because of no accurate SU level. Finally, cutaneous psoriasis extent has been described to be a hyperuricemia risk factor [41]. In our analyses of psoriasis skin and nail involvement, only 2 patients had erythrodermic psoriasis. Most patients no longer had psoriasis with PsA treatment or had mild psoriasis. In that context, even if psoriasis area and severity index score had not been collected, it would have been useless.

Our study has some limitations. The retrospective data limit its power. Our inclusion criteria could have overestimated the severity of PsA because the use of ICD-10 coding allowed us to include only hospitalized patients, thus implying Berkson's bias [42]. In the same way, the prevalence of hyperuricemia and gout may have been overestimated in our population because of the inclusion of patients with at least one SU sample. In addition, the absence of disease activity as a means of assessing remission in patients is a notable limitation. This is indeed due to the retrospective design of our study.

The main strength of our study is that it is the first to specifically evaluate PsA in terms of SU level. A large number of patients were included, for which we also had little missing data because of deep reading of medical files.

The present study allows for distinguishing 2 distinct PsA profiles according to their association with hyperuricemia (Fig. 1).

Our results support a potential worsening impact of MSU crystals on PsA, previously named psout [1]. The existence of this entity would help in the medical care of patients. In practice, a flare of PsA must be determined as not a gout attack. Beyond that, knowledge of hyperuricemic PsA should lead to even more personalized medicine.

Here, we propose recommendations for the diagnosis of psout and management of PsA with or without hyperuricemia (Fig. 2).

To confirm this viewpoint, new studies, especially prospective and interventional ones, could help better understand the role of hyperuricemia in PsA.

\section{Conclusion}

Patients with hyperuricemic PsA show poorer response to PsA treatment and more peripheral and destructive joint damage than do normo-uricemic patients.
Fig. 1 Description of normoand hyperuricemic psoriatic arthritis. CRF, moderate to severe chronic renal failure; MACEs, major adverse cardiovascular events; HBP, high blood pressure; MetS, metabolic syndrome; PsA, psoriatic arthritis

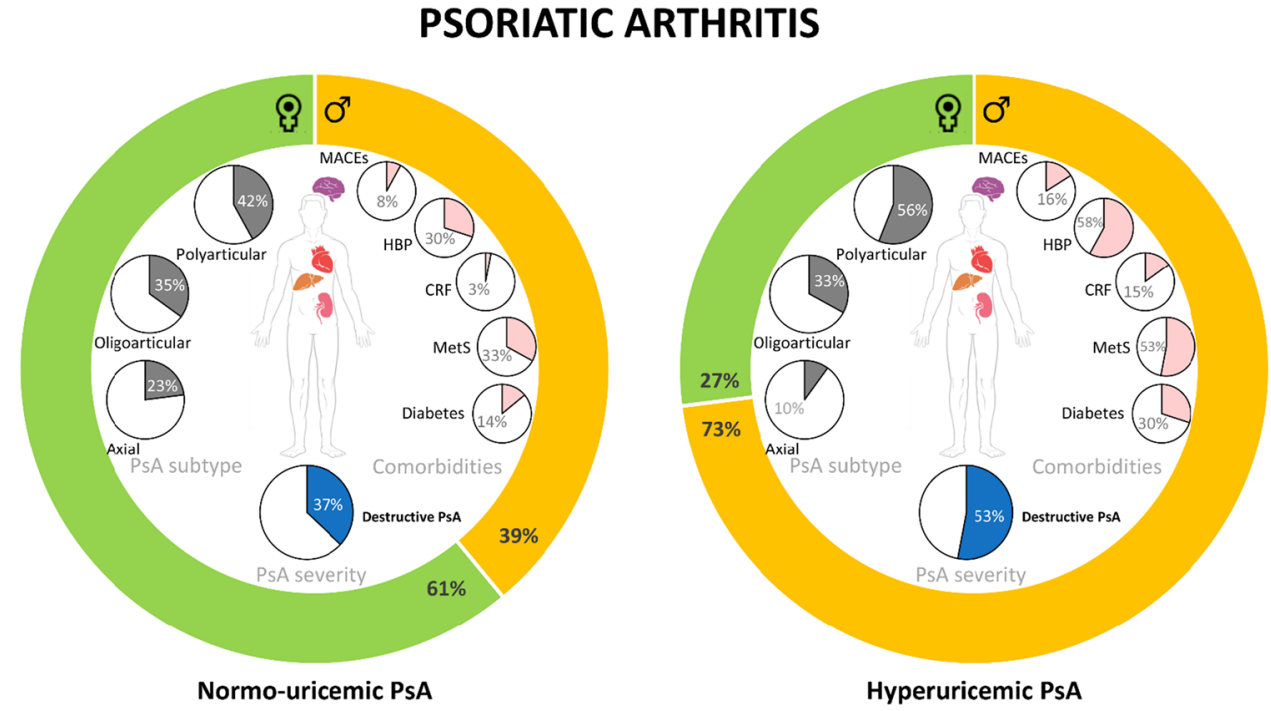

Springer 
Fig. 2 Recommendations for the diagnosis of psout and management of PsA with or without hyperuricemia. DECT, dual energy computed tomography; MSU, monosodium urate. Because of the retrospective method of our study, these recommendations should be taken with caution

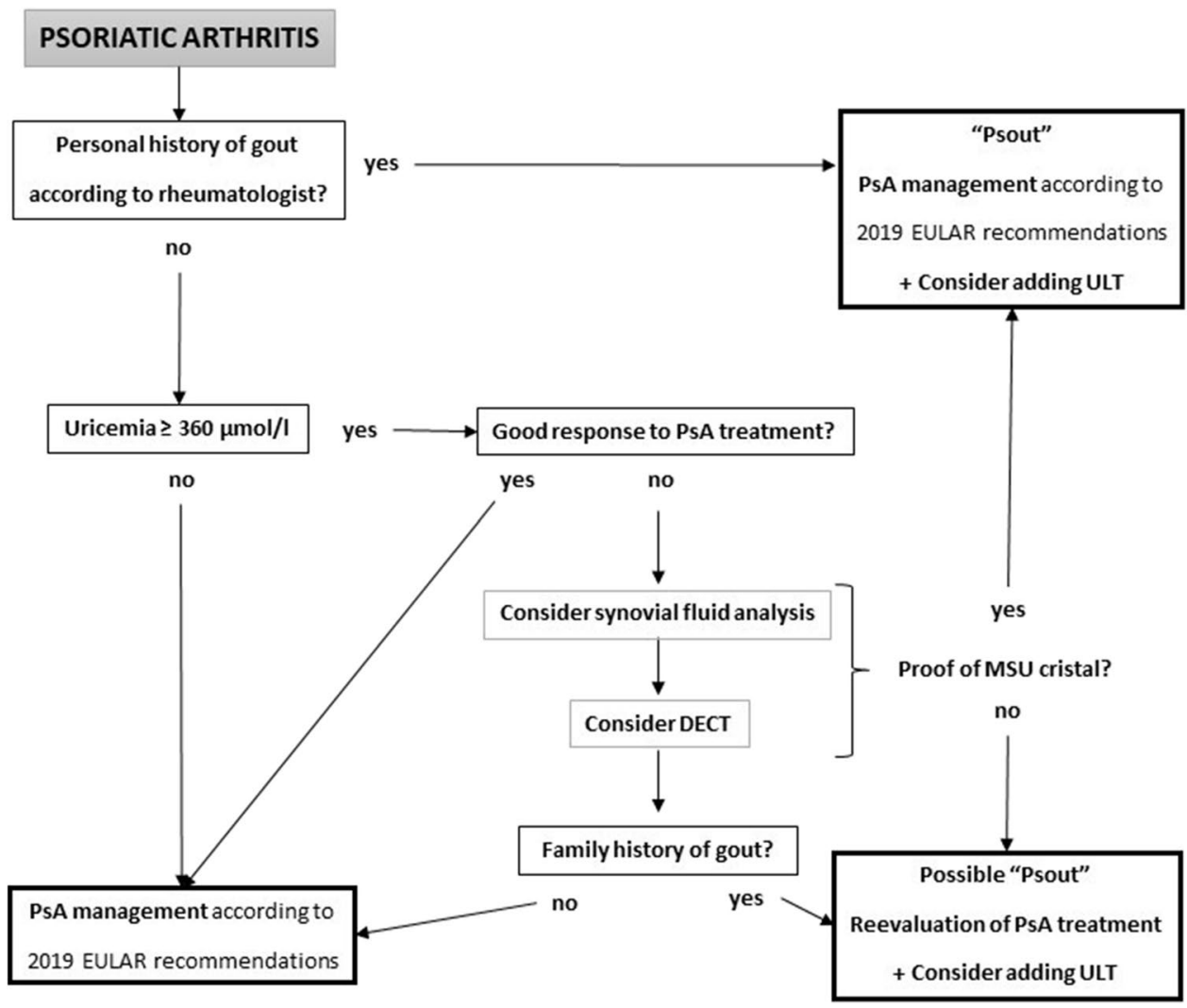

Acknowledgements We thank all participating patients. We also thank the medical secretaries for their help with the ICD-10 extraction and Dr. Thomas Lavaux for helping with serum urate tests at Strasbourg University Hospital.

Author contribution Dr. Laura Widawski was the principal investigator, had full access to all of the medical data, and takes responsibility for the integrity of the data collection and interpretation. Dr. Thibaut Fabacher performed all the statistical analyses. Dr. Renaud Felten and Dr. Laurent Messer supervised the study and helped design it with Dr. Pierre-Marie Duret and Dr. Lionel Spielmann. Pr Jacques-Eric Gottenberg and Pr Jean Sibilia codesigned the study and contributed to the interpretation of the results. All authors contributed to the critical revision of the manuscript for important intellectual content and approved the final version.

Data availability All data generated or analyzed during this study are included in this published article.

Code availability Not applicable.

\section{Declarations}

Ethics approval This study was validated on August 24, 2020, by the Strasbourg University Hospital Ethics Committee (CE-2020-134).

Patient involvement Patients were not involved in the design, conduct, reporting, and dissemination plans of this article.

Consent to participate Not applicable.

Consent for publication Not applicable.
Disclosures None.

Open Access This article is licensed under a Creative Commons Attribution 4.0 International License, which permits use, sharing, adaptation, distribution and reproduction in any medium or format, as long as you give appropriate credit to the original author(s) and the source, provide a link to the Creative Commons licence, and indicate if changes were made. The images or other third party material in this article are included in the article's Creative Commons licence, unless indicated otherwise in a credit line to the material. If material is not included in the article's Creative Commons licence and your intended use is not permitted by statutory regulation or exceeds the permitted use, you will need to obtain permission directly from the copyright holder. To view a copy of this licence, visit http://creativecommons.org/licenses/by/4.0/.

\section{References}

1. Felten R, Duret P-M, Gottenberg J-E, et al. At the crossroads of gout and psoriatic arthritis: 'psout'. Clin Rheumatol Published Online First: 15 February 2020.https://doi.org/10.1007/ s10067-020-04981-0

2. Ritchlin CT, Colbert RA, Gladman DD (2017) Psoriatic arthritis. N Engl J Med 376:957-970. https://doi.org/10.1056/NEJMra1505557

3. Barnett R (2018) Gout. The Lancet 391:2595. https://doi.org/ 10.1016/S0140-6736(18)31366-7

4. Costa NT, Scavuzzi BM, Iriyoda TMV et al (2018) Metabolic syndrome and the decreased levels of uric acid by leflunomide favor redox imbalance in patients with rheumatoid arthritis. Clin Exp Med 18:363-372. https://doi.org/10.1007/ s10238-018-0500-y 
5. AlJohani R, Polachek A, Ye JY et al (2018) Characteristic and outcome of psoriatic arthritis patients with hyperuricemia. J Rheumatol 45:213-217. https://doi.org/10.3899/jrheum.170384

6. Arevalo AB, Haddadin F, Contreras G et al (2019) Sat0371 Cardiovascular impact of hyperuricemia in patients with psoriatic arthritis. Ann Rheum Dis 78:1268-1268. https://doi.org/ 10.1136/annrheumdis-2019-eular.1674

7. Golov KG, Ivanov OL, Balkarov IM et al (1994) Clinical significance of hyperuricemia in psoriasis. Klin Med (Mosk) 72:34-36

8. Kuo C-F, Grainge MJ, Mallen C et al (2016) Comorbidities in patients with gout prior to and following diagnosis: casecontrol study. Ann Rheum Dis 75:210-217. https://doi.org/10. 1136/annrheumdis-2014-206410

9. Lai TL, Yim CW, Wong PY et al (2018) Hyperuricemia in Asian psoriatic arthritis patients. Int J Rheum Dis 21:843-849. https:// doi.org/10.1111/1756-185X.13265

10. Merola JF, Wu S, Han J, et al. Psoriasis, psoriatic arthritis, and risk of gout in U.S. men and women. Ann Rheum Dis 2015;74:1495500. https://doi.org/10.1136/annrheumdis-2014-205212

11. Pascart T, Latourte A, Chalès G et al (2020) Recommandations de la Société française de rhumatologie pour la prise en charge de la goutte: le traitement hypo-uricémiant. Rev Rhum 87:332-341. https://doi.org/10.1016/j.rhum.2020.07.009

12. FitzGerald JD, Dalbeth N, Mikuls T et al (2020) 2020 American College of Rheumatology Guideline for the Management of Gout. Arthritis Care Res (Hoboken) 72:744-760. https://doi.org/ 10.1002/acr.24180

13. Loeb JN (1972) The influence of temperature on the solubility of monosodium urate. Arthritis Rheum 15:189-192. https://doi.org/ 10.1002/art.1780150209

14. Bardin $\mathrm{T}$ (2015) Hyperuricemia starts at 360 micromoles $(6 \mathrm{mg} /$ dL). Joint Bone Spine 82:141-143. https://doi.org/10.1016/j. jbspin.2015.01.002

15. Neogi T, Jansen TLTA, Dalbeth N et al (2015) 2015 Gout classification criteria: an American College of Rheumatology/ European League Against Rheumatism collaborative initiative. Ann Rheum Dis 74:1789-1798. https://doi.org/10.1136/annrh eumdis-2015-208237

16. Hyndman D, Liu S, Miner JN. Urate handling in the human body. Curr Rheumatol Rep 2016; 18. https://doi.org/10.1007/ s11926-016-0587-7

17. Moll JMH, Wright V (1973) Psoriatic arthritis. Semin Arthritis Rheum 3:55-78. https://doi.org/10.1016/0049-0172(73)90035-8

18. Alberti KGMM, Zimmet P, Shaw J. Metabolic syndrome-a new world-wide definition. A consensus statement from the International Diabetes Federation. Diabetic Medicine 2006;23:469-80. https://doi.org/10.1111/j.1464-5491.2006.01858.x

19. Charlson M, Szatrowski TP, Peterson J et al (1994) Validation of a combined comorbidity index. J Clin Epidemiol 47:1245-1251. https://doi.org/10.1016/0895-4356(94)90129-5

20. Akaike H (1974) A new look at the statistical model identification. IEEE Trans Autom Control 19:716-723. https://doi.org/10.1109/ TAC.1974.1100705

21. Tsai Y-G, Chang D-M, Kuo S-Y et al (2003) Relationship between human lymphocyte antigen-B27 and clinical features of psoriatic arthritis. J Microbiol Immunol Infect 36:101-104

22. López-Larrea C, Torre Alonso JC, Rodriguez Perez A et al (1990) HLA antigens in psoriatic arthritis subtypes of a Spanish population. Ann Rheum Dis 49:318-319

23. Ogdie A, Schwartzman S, Husni M (2015) Recognizing and managing comorbidities in psoriatic arthritis. Curr Opin Rheumatol 27:118-126. https://doi.org/10.1097/BOR.0000000000000152

24. Catanoso MG, et al. FRI0350 Factors associated with peripheral erosive radiographic disease in a consecutive series of 794 psoriatic arthritis patients. ARD 2020.
25. Bardin T, Doherty M, et al. Estimation de la prévalence de la goutte en 2013 en France., M. Doherty et coll. 2013.

26. Burnett JW (1982) Acute gout secondary to psoriasis exacerbated by photochemotherapy. Arch Dermatol 118:211-211. https://doi. org/10.1001/archderm.1982.01650150073031

27. Liu M, Li J-H, Li B et al (2009) Coexisting gout, erythrodermic psoriasis and psoriatic arthritis. Eur J Dermatol 19:184-185. https://doi.org/10.1684/ejd.2008.0607

28. Lobato LC, Coutinho JC, Frota MZM et al (2017) Chronic tophaceous gout in patients with psoriasis. An Bras Dermatol 92:104106. https://doi.org/10.1590/abd1806-4841.20174895

29. Venkatasubramaniam KV, Bluhm GB, Riddle JM (1980) Psoriatic arthropathy and crystal-induced synovitis. J Rheumatol 7:213-217

30. Cosimo De Bari FPC Giovanni Lapadula (1998) Coexisting psoriatic arthritis, gout, and chondrocalcinosis: case report. Scand J Rheumatol 27:306-309. https://doi.org/10.1080/0300974984 42442

31. Morasiewicz P, Koprowski P, Dragan S (2014) Coexisting psoriasis, psoriatic arthritis, gout and Dupuytren's contracture. Ortop Traumatol Rehabil 16:441-447. https://doi.org/10.5604/15093 492.1119622

32. Bosmansky K, Trnavsky K (1983) Psoriasis and gout: report of 4 cases. Clin Rheumatol 2:423-426. https://doi.org/10.1007/bf020 41566

33. Kumar A. U. A, Browne LD, Li X, et al. Temporal trends in hyperuricaemia in the Irish health system from 2006-2014: A cohort study. PLoS One 2018;13. https://doi.org/10.1371/journal.pone. 0198197

34. Tsuruta N, Imafuku S, Narisawa Y (2017) Hyperuricemia is an independent risk factor for psoriatic arthritis in psoriatic patients. J Dermatol 44:1349-1352. https://doi.org/10.1111/1346-8138. 13968

35. Li S, Cui L, Cheng J et al (2020) Repeated measurements of serum urate and mortality: a prospective cohort study of 152,358 individuals over 8 years of follow-up. Arthritis Res Ther 22:84. https://doi.org/10.1186/s13075-020-02173-4

36. Kasl SV, Cobb S, Brooks GW (1968) Changes in serum uric acid and cholesterol levels in men undergoing job loss. JAMA 206:1500-1507. https://doi.org/10.1001/jama.1968.0315007003 8006

37. Zitt E, Fischer A, Lhotta K et al (2020) Sex- and age-specific variations, temporal trends and metabolic determinants of serum uric acid concentrations in a large population-based Austrian cohort. Sci Rep 10:7578. https://doi.org/10.1038/s41598-020-64587-z

38. Maclachlan MJ, Rodnan GP (1967) Effects of food, fast and alcohol on serum uric acid and acute attacks of gout. Am J Med 42:38-57. https://doi.org/10.1016/0002-9343(67)90005-8

39. Kang MW, Chin HJ, Joo K-W et al (2019) Hyperuricemia is associated with acute kidney injury and all-cause mortality in hospitalized patients. Nephrology 24:718-724. https://doi.org/10.1111/ nep. 13559

40. Vargas-Santos AB, Neogi T (2017) Management of gout and hyperuricemia in CKD. Am J Kidney Dis 70:422-439. https:// doi.org/10.1053/j.ajkd.2017.01.055

41. Li X, Miao X, Wang H, et al. Association of serum uric acid levels in psoriasis. Medicine (Baltimore) 2016;95. https://doi.org/10. 1097/MD.0000000000003676

42. Berkson J (2014) Limitations of the application of fourfold table analysis to hospital data. Int J Epidemiol 43:511-515. https://doi. org/10.1093/ije/dyu022

Publisher's note Springer Nature remains neutral with regard to jurisdictional claims in published maps and institutional affiliations. 